\title{
EDITORIAL
}

\section{“Impact of Research Conducted by Dr Ram B Singh on Modern Concepts of Nutrition \& Lifestyle in Mind-Body Diseases"}

For those who are acquainted with Dr Ram B Singh and his lifetime achievement dedicated to the creation of a family hospital in little Moradabad, India, and his research work, may conclude that there are differences between East and West research practices in medicine and that in some important areas these need to be bridged. Take for example, the one major conception of the 20th century which was more about cholesterol and saturated fats than about omega-3 fatty acids. Isn't it we, in the West, who have developed a capitalistic primary prevention approach to tackle what is at most a secondary risk factor? The West should perhaps critically question its own science and peer-review system before deliberately waiving its doubts about research in the East. There is room for humility and fierce resolve in the Western world. At the dawn of the 21 st century, the TsimTsoum Concept militates these two perspectives under the wise direction of Dr Ram B Singh whose vision and wisdom has been, and is, exemplary. We have a lot to learn from each other, from West to East. Perhaps India is at an appropriate geographical, historical and cultural place to facilitate communication and to help humanity progress. Hopefully Dr Ram B Singh will spread his influence so that as scientists we move on altogether into the 21 st century, provided there is enlightenment.

\section{INTRODUCTION}

The study of evolution of human beings indicates that man's early diet included naturally-occurring foods such as fruit, vegetables, greens, seeds, eggs and honey, fish and wild meat. That seasonally fluctuating food basket composition actually shaped modern human's genetic nutritional requirements. Refined cereal grains and extracted plant fats rich in trans and omega- 6 fatty acids are relatively recent additions to the human diet. They represent dramatic departure from the original dietary standards of the pre-refined food era. Early man also engaged in much physical activity with relatively minor mental stress.

- The potential role of diet in the prevention and pathogenesis of cardiovascular disease (CVD), type-2 diabetes and insulin resistance is largely described in the medical-related literature. Excess energy intake, trans fatty acids (TFA), refined starches and sugar are pro-inflammatory. Because they are essential, excess of omega- 6 fatty acids associated with real or apparent deficiencies in omega-3 fatty acids is of primary importance in the pathogenesis of the metabolic syndrome leading to CVD.

- Sedentary behavior in conjunction with mental stress and various modern socio-psychological and personality traits enhance sympathetic activity and increase secretion of catecholamines, cortisol and serotonin, which through underlying mechanisms appear to fuel inflammation and instigate metabolic syndrome.

- The synergistic action of inappropriate diet and lifestyle via excess release of otherwise regulatory hormones and hormonelike mediators, especially during fetal life, infancy and childhood, result in dysfunction of neurons and receptors later in life.

Blood lipid composition does reflect one's health status wrt diet and lifestyle: (a) circulating serum lipoproteins and their ratio, provide information on their atherogenicity to blood vessels and (b) circulating plasma fatty acids, such as omega-6/3 fatty acid ratio, give an indication of the pro-inflammatory status of blood vessels; (a) and (b) are phenotype-related and depend on genetic, environmental and developmental factors. As such, they appear as universal markers for holistic health (Fig. 1). 


\section{ENERGY INTAKE/EXPENDITURE}

It would sound rather obvious to anyone, including the more intelligent layman, that food energy intake should in principle not exceed energy expenditure in an average 24-hr cycle. Given the number of overweight and obese subjects in Western and other populations, one must however reconsider the meaning of the term 'obvious', if only for the sake of not offending individuals. Something else must indeed come into play to explain the apparent unavoidable trend of increased weight with age; a trend that appears to take off ever-earlier in the process of ageing over the past 100-150 years. Food over-availability is commonly thought of as a basic cause of this epidemic. However, animals in the wild, irrespective of whether they be herbivorous or carnivorous, as well as tamed farm animals do not become over-weight when fed ad libitum, naturally or domestically. Obesity is a characteristic of humans, including their humanized animal companions, viz. pets. In this respect, it becomes seemingly obvious that obesity is a mind-related disease that leads to premature body decay.

\section{LIFESTYLE}

Body Mass Index (BMI) and Body Mass Composition (BMC) are generally used for assessing quality standards for nutrition and lifestyle. At constant body weight, aging translates into the substitution of lean- for fat-mass. The process is associated with the progressive increase in blood concentrations of a large number of biomarkers for pre-metabolic syndrome, including cholesterol. Changes in body mass composition at constant weight therefore appears to be body-related and in fact naturally occurs in the wild as well.

\section{From bad w6-LDL-C to good w6:w3 -LDL-C}

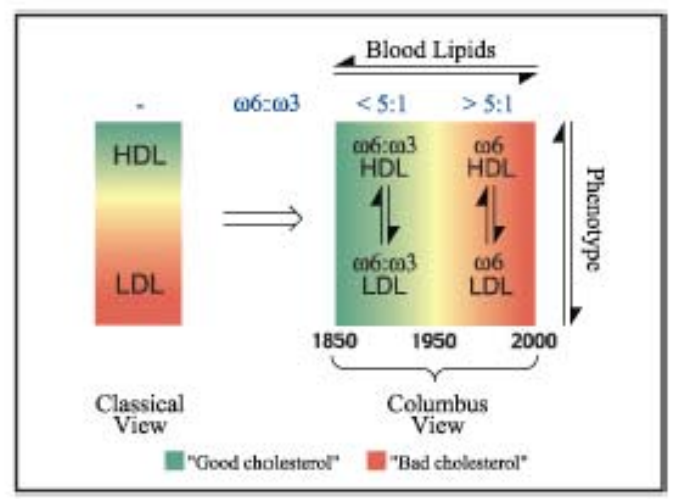

Fig.1. From bad $\omega 6$-LDL-C to good $\omega 6$ : $\omega 3$-LDL-C. The classical description of good HDL and bad LDL cholesterol may well be holding true in our modern societies for only very little attention has been drawn to the $\omega 6: \omega 3$ ratio, and this may well explain the apparently contradicting results published over the last 50 years in the literature and used overly and partially by some to try demonstrate the classical diet-heart relationship. In fact, the HDL:LDL balance is mostly phenotype-driven and it might not be necessarily safe to try change this individual equilibrium in one way or another by applying currently proposed dietary and/or medical treatments. On the contrary, changing dietary lipid pattern and, so-doing, returning blood essential fatty acids to what our genetic profile expects them to be would probably help a much longer way in keeping us heart healthy.

\section{CHOLESTEROL, SATURATED AND MONO-UNSATURATED FATS}

Dietary cholesterol, saturated and mono-unsaturated fats are non-essential to humans and it therefore does not seemingly matter whether these nutrients are substantially present or absent. The cornucopia of studies and articles published in the scientific literature demonstrate the obvious fact that a non-essential nutrient can not represent a primary risk factor for diseases be what may, yet may well represent a secondary risk factor of relative importance when one or more essential nutrients are misrepresented or, more simply, when the energy intake/expenditure is out of balance. 


\section{OMEGA-6/3 FATTY ACIDS}

Omega-6/3 fatty acids are essential to humans and they compete for similar metabolic pathways of importance in thermodynamic aspects (homeostasis) of health. It is recognized that the past 100-150 years of agro-industrial revolution have induced major changes in the distribution of these two competing families of fatty acids in the human diet, leading to oversaturation of fatty acid metabolic pathways by the omega- 6 series. In turn, inflammatory indexes have jumped and become markers of pre-metabolic syndrome at an ever-decreasing early age to the extent that it is now thought that chronic degenerative diseases may in fact pass to the next generation through epigenetic modifications. The case of agouti mice may serve as an example of how nutrients may indeed guide inheritance.

\section{EVOLUTION}

Theories of evolution are many, but most, if not all, scientists would go well along with the present common sense that life on earth, including humans, evolved from the early pre-biotic chemistry that began billions years ago. There seems to be no end to such changes as time passes by and environmental conditions change at such a slow pace to allow all possible reversible chemical processes to take place. Natural/cultural selection does the rest.

In this respect, the recently man-induced double changes in environmental conditions, i.e. (1) stress-strain time window and (2) omega-6/3 fatty acids, may come to select a new group of degenerated humans.

\section{CONCLUSION}

For the first time in history, one assists an evolutionary process all set up by man that appears to give selective advantage to overweight, i.e. obese type-A behavior individuals. Modern sedentary stressful lifestyles, in conjunction with high omega-6/3 diets, pave the way to a new species of degenerated humans to take the lead. Cholesterol and saturated fats will not solve the problem. The work of Dr R. B. Singh, inter alia, in recognizing the contributions which East and West can make together in the quest for improved health for all is to be applauded.

\section{FABIEN DE MEESTER}

SEP 28, 2010 